\title{
EFFECTIVENESS OF UV LIGHT AS A MEANS TO REDUCE SALMONELLA CONTAMINATION ON TOMATOES AND FOOD CONTACT SURFACES
}

\author{
Winnie Lim and Mark A. Harrison*
}

Running title: UV treatment to reduce Salmonella contamination on tomatoes

*Corresponding author:

Department of Food Science and Technology

University of Georgia

Athens, GA 30602

(706) $542-1088$

FAX (706) 542-1050

mahfst@uga.edu 
1

2

3

4

5

6

7

8

9

10

11

12

\section{Abstract}

The effectiveness of ultraviolet light at a wavelength of $254 \mathrm{~nm}$ to reduce Salmonella contamination on tomatoes and food contact surfaces was evaluated. Inoculated tomatoes were exposed to UV-C light at doses ranging from 0 to $223.1 \mathrm{~mJ} / \mathrm{cm}^{2}$. All UV treatments significantly reduced Salmonella populations $(\mathrm{p}<0.05)$. The effectiveness of UV-C light in reducing Salmonella contamination on different locations on tomato surfaces under various UV doses (0$117.2 \mathrm{~mJ} / \mathrm{cm}^{2}$ ) was also explored. Results indicated that regardless of the locations, UV treatment was shown to be effective in decreasing Salmonella populations. Subsequent studies evaluated possible photoreactivation or dark repair of injured Salmonella post-UV treatment. Following UV light exposure at doses of 0, 22.3, 44.6, and $89.2 \mathrm{~mJ} / \mathrm{cm}^{2}$, tomatoes were either exposed to visible light for 0,3 , and 5 h or stored in the dark for the same amount of time. Photoreactivation was not detected, nor was dark repair. UV light was also evaluated for its effectiveness to reduce Salmonella contamination on food contact surfaces (stainless steel, HDPE, waxed cardboard and PVC). Contaminated coupons were exposed to UV-C light at 0, 3.3, and $19.7 \mathrm{~mJ} / \mathrm{cm}^{2}$. Significant differences were observed between coupons treated with UV light and the controls $(\mathrm{p}<0.05)$. Coupons exposed for longer time had greater Salmonella population reductions, except for waxed cardboard coupons. Application of UV-C light to reduce Salmonella contamination in tomato handling facilities is feasible.

Keywords: UV-C light, Salmonella, tomatoes, food contact surfaces 


\section{Introduction}

The frequency of reported foodborne illness outbreaks linked to fresh produce has increased in recent decades. This increase may be due to increased surveillance and increased consumption of fresh produce (Doyle \& Erickson, 2008). Since most fresh produce is consumed raw or minimally processed, pathogen contamination may be a potential threat to human health. From 1990 to 2005, produce outbreaks were responsible for $13 \%$ of all foodborne outbreaks and 21\% of all foodborne illnesses (DeWaal \& Bhuiya, 2007). A more recent report, analyzing outbreaks from 1998-2007, indicated that produce outbreaks caused 15\% of all foodborne outbreaks and 23\% of all foodborne illnesses (Center for Science in the Public Interest, 2009).ESPI). Most of the produce-associated outbreaks and illnesses were associated with greens-based salads, cantaloupes, tomatoes, lettuce, and sprouts (Olaimat\& Holley, 2012). Salmonella is one of the primary pathogens associated with produce outbreaks and accounts for 17\% of the produce outbreaks during 1998 and 2007 (Center for Science in the Public Interest, 2009).ESPI). Produce items associated with outbreaks of salmonellosis include sprouts, greensbased salad, melon, and potatoes (DeWaal \& Bhuiya, 2007).

The first large multistate Salmonella outbreaks related to tomato consumption were reported back in 1990 (Hedberg et al., 1999). The tomato contamination source implicated in at least two outbreaks, involving Salmonella Javiana and Salmonella Montevideo, was traced to tomato packinghouses (CDC, 2005). Results of a study done by Allen et al., (2005) indicated that Salmonella can survive on tomato surfaces and packing line surfaces under common environmental conditions.

Interventions are needed to minimize pathogen contamination of raw produce and to eliminate them if present on produce. Normally, fresh produce receives little microbial 
intervention other than washing. For these reasons, alternative strategies to decrease pathogenic bacterial levels on fresh produce are needed. One of the alternatives is to use ultraviolet-C (UVC) light at $254 \mathrm{~nm}$.

UV light is used for air, water/liquid, and surface decontamination treatments (Bintsis et al., 2000). Its application has been limited due to the poor penetration capabilities of the UV wavelengths and shadowing effects (Sizer \& Balasubramaniam, 1999). UV’s effectiveness to reduce bacterial contamination on produce has been documented (Chun et al., 2010; Escalona et al., 2010; Sommers et al., 2010; Yaun et al., 2004). Mukhopadhyay et al., (2013) found that UV in combination with low dose gamma irradiation doses resulted in $<4 \log$ reduction of $E$. coli and Salmonella on grape tomatoes. UV light is also effective in reducing black mold, gray mold, and Rhizopus soft rot on tomatoes. Treated tomatoes were firmer and had slower ripening, indicating extended shelf life (Lui et al., 1993). UV light offers some other advantages as it does not leave any residue, it does not have legal restrictions, it does not require installation of extensive safety equipment (Wong et al., 1998; Yousef \& Marth, 1988), it is easy to use, and it is economical (Bintisis et al., 2000). Microbial inactivation is caused by the cross-linking of pyrimidine dimers in DNA which prevents microbial reproduction (Sizer \& Balasubramaniam, 1999). However, many microorganisms have developed mechanisms to repair the UV-induced DNA damage. One of the repair mechanisms is photoreactivation. Photoreactivation is a lightdependent process which involves photolyase to reverse UV-induced DNA damage (Harm, 1980). Therefore, photoreactivation increases the possibility that microorganisms might regain viability after UV light treatment and thus raise food safety concerns. Photoreactivation of fecal coliforms and E. coli has been reported (Hallmich \& Gehr, 2010; Sommer et al., 2000; Tosa \& Hirata, 1999; Zimmer \& Slawson, 2002). Hu et al., (2012) found that Salmonella Typhimurium, 
Shigella dysenteriae, and E. coli are able to photoreactivate after UV treatment. Meanwhile, Kuo et al., (1997) did not notice photoreactivation of $S$. Typhimurium on shell eggs.

There are some uncertainties concerning the effectiveness of UV light on reducing Salmonella contamination on mature green tomatoes and whether photoreactivation of UVinjured Salmonella on tomatoes occurs. If UV light is applied to tomatoes in a processing line, it is likely that not all spots on the tomatoes will be directly exposed to the UV light as tomatoes will be rolling on the conveyer belt. Thus, effectiveness of UV-C light in reducing Salmonella contamination on different locations on tomato surfaces needs to be determined. In addition, efficacy of UV light in reducing Salmonella contamination on food contact surfaces commonly encountered in tomato handling facilities is also of interest. Thus, the objectives of this study were: (1) to determine the effectiveness of UV-C light in reducing Salmonella populations on tomatoes; (2) to evaluate the effectiveness of the treatment to reduce Salmonella contamination regardless of its location on the tomato surface; (3) to determine whether photoreactivation by the visible light or the dark repair mechanisms can result in the recovery of damaged Salmonella cells post-UV treatment; and (4) to study the effectiveness of UV light to decrease Salmonella contamination on food contact surfaces that could be used in tomato handling facilities.

\section{Materials and Methods}

\subsection{Bacterial Cultures and Growth Media}

Rifampicin resistant Salmonella enterica serovars Michigan_(cantaloupe isolate), Montevideo (human isolate from a tomato outbreak), Newport (environmental isolate from a Virginia tomato outbreak), Poona (human isolate from a cantaloupe outbreak), and Saintpaul (orange juice isolate) were obtained from the Citrus Research and Education Center, University of Florida, Lake Alfred, FL (Table 1)and were adapted to $100 \mu \mathrm{g} / \mathrm{ml}$ of rifampicin. All strains 
were preserved on Microbank beads (Microbank; Pro-Lab Diagnostics, Austin, TX) at $-80^{\circ} \mathrm{C}$. Prior to use, each strain was activated by three successive transfers into 9 ml tryptic soy broth (Becton, Dickinson and Company, Sparks, MD) containing $100 \mu \mathrm{g} / \mathrm{ml}$ of rifampicin (Cat. \# BP2679-5; Fisher Scientific, Pittsburgh, PA) for $24 \mathrm{~h}$ at $35^{\circ} \mathrm{C}$. Rifampicin stock solution was prepared by dissolving $1 \mathrm{~g}$ of rifampicin in $20 \mathrm{ml}$ of methanol (Cat. \# A412SK-4; Fisher Scientific, Pittsburgh, PA) and filtered through $0.22 \mu \mathrm{m}$ Millipore Express ${ }^{\circledR}$ PLUS Membrane (Cat. \# SCGP00525; Millipore Corporation, Billerica, MA).

On the day of the experiment, $2 \mathrm{ml}$ of each Salmonella serovar were combined to produce a $10 \mathrm{ml}$ Salmonella cocktail. The $10 \mathrm{ml}$ Salmonella cocktail was centrifuged for $10 \mathrm{~min}$ at a relative centrifugal force (RCF) of 2,500 x g (Model 5681; Forma Scientific, Inc., Marietta, OH) and the supernatant was decanted. The pellet was washed twice with $10 \mathrm{ml}$ of $0.1 \%$ peptone water (Becton, Dickinson and Company, Sparks, MD) and resuspended in $10 \mathrm{ml}$ of $0.1 \%$ peptone water for final use. Tryptic soy agar (TSA; Becton, Dickinson and Company, Sparks, MD) with $100 \mu \mathrm{g} / \mathrm{ml}$ rifampicin (TSAR) was used to enumerate Salmonella. Resuspended cultures were serially diluted with $0.1 \%$ peptone water and enumerated to verify the initial concentration. Resuspended cultures contained approximately $10^{8}-10^{9} \mathrm{CFU} / \mathrm{ml}$ Salmonella.

\subsection{UV-C Light Source}

The UV-C light source (American Air \& Water, Inc.; Hilton Head Island, SC) was a closed end reflector (CE) germicidal fixture, CE-36-2, with two germicidal (254 nm) slim line (GML005) lamps. The dimension of the CE fixture is $94 \times 18 \times 19 \mathrm{~cm}$. The UV lamp fixture was mounted horizontally by adjustable, tripod style legs. The UV-C lamp apparatus and exposure area was enclosed with sidings lined with aluminum foil to increase the containment of the UV light. The surface on which the samples were placed was covered with bench coat (Versi-Dry 
Lab Soakers; Fisher Scientific, Pittsburgh, PA). The intensity of the UV-C light was measured using a Newport power meter model 1928-C (Newport Corporation, Irvine, CA). The UV-C light was allowed to warm up at least 30 min prior to use.

\subsection{Effectiveness of UV-C Light (254 nm) in Reducing Salmonella Contamination on} Tomatoes under Varying UV-C Doses

Mature, unwaxed green tomatoes were obtained from a local distributor. Tomatoes were stored at $4^{\circ} \mathrm{C}$ until used and allowed to equilibrate to room temperature for approximately $1 \mathrm{~h}$ before inoculation. Tomatoes were spot inoculated_Beuchat et al. 2001) with $100 \mu \mathrm{l}$ of Salmonella cocktail within marked areas $\left(\sim 6 \mathrm{~cm}^{2}\right)$ on the equatorial plane of each tomato. After inoculating, tomatoes were air-dried for approximately $2 \mathrm{~h}$ in a biological safety cabinet before exposing to UV-C light. Positive control tomatoes which were not UV treated were sampled to determine the initial Salmonella inoculation level. Negative control tomatoes were not inoculated with the Salmonella cocktail.

Tomatoes were placed so the inoculated areas were directly exposed to UV light for 0 , $30,60,90,120,150,180,210,240,270$, and $300 \mathrm{~s}$. The average intensity of the UV light was $743.6 \mu \mathrm{W} / \mathrm{cm}^{2}$. The corresponding doses were $0,22.3,44.6,66.9,89.2,111.5,133.9,156.1$, 178.5, 200.8, and $223.1 \mathrm{~mJ} / \mathrm{cm}^{2}$. The distance between the center of the light and top of tomato was approximately $53.3 \mathrm{~cm}$.

Treated tomatoes were placed in individual sterile stomacher bags containing $20 \mathrm{ml}$ of universal preenrichment broth (Becton, Dickinson and Company, Sparks, MD). Tomatoes were manually shaken for $30 \mathrm{~s}$, hand-rubbed for $30 \mathrm{~s}$ at the inoculation spot, and shaken for another 30 s. Serial dilutions were made using $9 \mathrm{ml}$ of universal preenrichment broth and dilutions were spiral plated (Autoplate 4000; Spiral Biotech, Inc., Bethesda, MD) onto TSAR. The detection 
limit was $200 \mathrm{CFU} / \mathrm{ml}$. All plates were incubated at $35^{\circ} \mathrm{C}$ for $24 \mathrm{~h}$ before enumerating surviving Salmonella. Negative control tomatoes were plated on both TSA and TSAR plates to check for background microflora.

\subsection{Evaluation of Whether the Orientation of Salmonella Contaminated Tomatoes Influences the Effectiveness of UV-C Light}

Inoculation of mature green tomatoes was the same as described previously. Six tomatoes with Salmonella inoculated on the same spot of tomato surface, were positioned differently under UV-C light to evaluate the effectiveness of the treatment to reduce Salmonella contamination regardless of its location on the tomato surface (whether it was directly or indirectly exposed to the UV light) for each replication (Fig. 1). The average intensity of the UV light at those 6 spots was $651 \mu \mathrm{W} / \mathrm{cm}^{2}$. Tomatoes were exposed at doses of $0,19.5,39.1,58.6,78.1,97.6$, and 117.2 $\mathrm{mJ} / \mathrm{cm}^{2}$ for $0,30,60,90,120,150$, and $180 \mathrm{~s}$. The distance between the center of the light and the tomato surface was kept the same as in the previous study. Recovery of Salmonella from tomatoes was done in the same manner as mentioned previously, except that dilutions were spread plated instead of spiral plated.

\subsection{Evaluation of Possible Photoreactivation of Injured Salmonella Post-UV-C}

\section{Treatments}

This study determined the possibility of repair of injured Salmonella post-UV-C treatments with visible light. Inoculated mature green tomatoes were exposed directly to UV-C light at various doses of $0,22.3,44.6$, and $89.2 \mathrm{~mJ} / \mathrm{cm}^{2}$ for $0,30,60$, and $120 \mathrm{~s}$ at a distance of approximately $53.3 \mathrm{~cm}$ from the tomato surface. Following UV-C exposure, tomatoes were exposed to visible light from 8 fluorescent lamps in a chamber (Percival model E-41 HO; Percival Scientific, Inc., Perry, IA). The intensity of the visible light was approximately 10 
$\mathrm{mW} / \mathrm{cm}^{2}$ measured with Newport power meter model 1928-C. The inoculated tomatoes were exposed to the lamps for 0,3 , and $5 \mathrm{~h}$. The distance between the light source and tomato surface was approximately $43.8 \mathrm{~cm}$. The temperature of the chamber was $20^{\circ} \mathrm{C}\left( \pm 0.5^{\circ} \mathrm{C}\right)$.

To determine if recovery under darkness occurred, inoculated tomatoes treated after UV$\mathrm{C}$ light treatment were stored in the dark in stainless steel containers for 0,3 , and $5 \mathrm{~h}$ at room temperature, $19-20.5^{\circ} \mathrm{C}$. Subsequent to the respective visible light and dark exposure, treated tomatoes were placed in individual sterile stomacher bags containing $20 \mathrm{ml}$ of universal preenrichment broth and sampled for surviving Salmonella as previously described.

\subsection{Effectiveness of UV-C light ( $254 \mathrm{~nm})$ in Reducing Salmonella Contamination on} Food Contact Surfaces under Varying UV-C Doses

Four food contact surfaces (stainless steel, high density polyethylene, polyvinyl chloride, and waxed cardboard) were chosen to simulate the contact surfaces that could commonly be used in commercial tomato facilities. Stainless steel coupons (SS; type 304, finish \#4B, $0.9 \mathrm{~mm}$ thick) sized (30 x $50 \mathrm{~mm}$ ) with smooth edges were fabricated by University of Georgia Instrument Shop, Athens, GA. Stainless steel coupons were autoclaved before use. High density polyethylene (HDPE; 2 mm thick; United States Plastic Corporation, Lima, OH) and polyvinyl chloride (PVC) belting (3 mm thick; \# 120 white; W.L. Deckert Company, Inc., Milwaukee, WI) were cut to size ( 30 x $50 \mathrm{~mm}$ ) using scissors and a utility knife, respectively. The cut coupons were used one time and autoclaved before use. Waxed cardboard (WC; 4 mm thick; International Paper Company, Griffin, GA) was cut to size (30 x $50 \mathrm{~mm}$ ) using a scalpel. Waxed cardboard coupons were sterilized by spraying 70\% ethanol (Decon Laboratories, Inc., King of Prussia, PA) over the front and back sides of the cardboard pieces. After 30 s exposure, the ethanol was 
wiped from coupon surfaces using a sterile Whirl-PaK ${ }^{\circledR}$ Speci-Sponge ${ }^{\circledR}$ Environmental Surface Sampling Sponge (38 x 76 mm; Nasco, Fort Atkinson, WI) wetted with sterile DI water.

All four types of coupons were placed in sterile metal pans and the surfaces were spot inoculated with $100 \mu \mathrm{l}$ of the resuspended Salmonella cocktail. Inoculated coupons were airdried in a biological safety cabinet for 90 min. Positive UV-control coupons did not receive UVC treatments. Negative control coupons were not inoculated. Three inoculated coupons of each type of surface were aseptically placed under UV-C light and exposed to UV-C light at an intensity of $656 \mu \mathrm{W} / \mathrm{cm}^{2}$ for $0,5\left(3.3 \mathrm{~mJ} / \mathrm{cm}^{2}\right)$ and $30 \mathrm{~s}\left(19.7 \mathrm{~mJ} / \mathrm{cm}^{2}\right)$ at a distance of $61.6 \mathrm{~cm}$. The $5 \mathrm{~s}$ and $30 \mathrm{~s}$ treatments were done on separate days.

After the UV treatment, treated coupons were aseptically placed in individual sterile stomacher bags containing $20 \mathrm{ml}$ of universal preenrichment broth. Treated coupons were handrubbed for $30 \mathrm{~s}$. Serial dilutions were made using $9 \mathrm{ml}$ of universal preenrichment broth and dilutions were spread plated onto TSAR. All plates were incubated at $35^{\circ} \mathrm{C}$ for $24 \mathrm{~h}$. Positive UV-control coupons were plated to determine initial concentration of Salmonella cocktail on coupons, and negative control coupons were also plated on both TSA and TSAR plates to check for background microflora.

\subsection{Statistical Analysis}

Experiments with tomatoes and food contact surfaces were replicated three times, with the exception of the first experiment, in which 8 repetitions were performed. Survival of Salmonella (CFU/tomato or CFU/coupon) was converted to $\log _{10}$. Statistical analysis was performed using SAS 9.3 (SAS Institute, Inc., Cary, NC). Analysis of variance was carried out by the general linear models (GLM) procedure. When differences among treatments existed $(p<0.05)$, Tukey's multiple comparison method was used to determine the difference. 


\section{Results and Discussion}

\subsection{Effect of UV-C Light in Reducing Salmonella Contamination on Tomatoes}

The efficacy of UV-C light in inactivating Salmonella on green tomatoes was evaluated. Salmonella populations inoculated on tomatoes significantly decreased $(\mathrm{p}<0.05)$ by 3.22 logs after UV treatment for $30 \mathrm{~s}\left(22.3 \mathrm{~mJ} / \mathrm{cm}^{2}\right)$. A maximum reduction of 4.39 log CFU/tomato was obtained when inoculated tomatoes were exposed for $240 \mathrm{~s}$, a UV dose of $178.5 \mathrm{~mJ} / \mathrm{cm}^{2}$ (Fig. 24). Thirty seconds and sixty seconds of exposure were not significantly different from each other. After 90 s of exposure, there was no further significant reduction. No Salmonella was detected from the negative controls, indicating the reductions noted were those of the challenge inoculum. Doyle and Erickson (2008) stated that interventions applied to produce should inactivate enteric pathogens by at least 3 logs. The results in this study showed that a 3 log reduction was achieved even with the lowest UV dose. Therefore, UV light can be an alternative intervention to inactivate pathogens on produce.

In a study by Yaun et al., (2004), the populations of Salmonella on inoculated tomatoes, which were obtained from a local distributor, were reduced by 2.19 log CFU/tomato when a maximum UV-C dose of $24 \mathrm{~mJ} / \mathrm{cm}^{2}$ was applied. Sommers et al., (2010) reported that Salmonella spp. inoculated on the surface of Roma tomatoes (maturity level not mentioned) were inactivated by $3.1 \log$ CFU/g at a dose of $500 \mathrm{~mJ} / \mathrm{cm}^{2}$. A higher $\log$ reduction, $3.8 \log \mathrm{CFU} / \mathrm{g}$, was obtained when UV-C dose of $4,000 \mathrm{~mJ} / \mathrm{cm}^{2}$ was used. The authors stated that the higher log reductions were below the detection limit. Song et al., (2011) found that UV-C at $5 \mathrm{~kJ} / \mathrm{m}^{2}(500$ $\mathrm{mJ} / \mathrm{cm}^{2}$ ) significantly decreased $S$. Typhimurium on light red cherry tomatoes by 2.58 logs. Yun et al., (2013) reported an initial decrease of 1 to 2 logs of E. coli O157:H7 and Salmonella on contaminated apricots after treatment with UV-C and noted that surviving populations decreased 
at a faster rate than non-UV exposed populations. Bermudez-Aguire \& Barbosa-Canovas (2013) found that inactivation of $E$. coli varied depending on the type of contaminated produce that was exposed to UV-C with the greatest reduction noted on the smooth surface of tomatoes. Williams et al., (2012) showed that combining pulsed UV treatments with sanitizers enhanced the reduction in Salmonella contamination on tomatoes. Hadjok et al. (2008) used UV light in combination with hydrogen peroxide to reduce Salmonella on fresh produce surfaces by at least

\section{4 logs and internalized Salmonella by at least 2.8 logs.}

Differences in the log reductions between this study and others may be due to using different strains of pathogens with varying levels of UV-sensitivity and UV-C sources of varying efficiencies. Shechmeister (1976) noted that UV sensitivities of bacteria vary not only among species, but also among strains of the same species. In addition, in some cases, the initial inoculum levels were not large enough to obtain greater log reduction calculations than those reported after UV treatment. For example, Sommers et al., (2010) obtained greater than 3 a Salmonella-log CFU reduction of Salmonella on tomatoes $3.08 \mathrm{CFU} / \mathrm{g}$ when exposed to UV at a dose of $500 \mathrm{~mJ} / \mathrm{cm}^{2}$. When, but even when a four-fold greater dose was applied, there was no significant increase in the log reduction. This study demonstrates that UV light had great efficiency in reducing Salmonella populations on tomatoes. The effectiveness of UV-C in reducing microbial growth in baby spinach, lettuce, melon, and fresh-cut apple has also been reported (Allende \& Artes, 2003; Allende et al., 2006; Escalona et al., 2010; Manzocco et al., 2011a \& Manzocco et al., 2011b).

\subsection{Orientation of Salmonella Contaminated Tomatoes under UV-C Light} Statistically, there was no difference in the level of Salmonella reduction regardless of interaction between the-UV treatment and the locations of Salmonella on tomato surfaces, 
meaning that UV at all tested dosesa certain dose exhibited the same statistical pattern regardless of Salmonella locations on tomatoes-and vice versa. After UV exposure, Salmonella populations were reduced $(\mathrm{p}<0.05)$ to similar levels regardless of the location of the Salmonella contamination on the tomato surface (Fig. $\underline{3} z)$ ). There was no significant difference in population reduction for the 30-120 s of exposures. While a similar level of Salmonella inactivation was obtained for the various contamination locations on the tomatoes, the population on position 1, facing directly to the UV light source, was reduced significantly more than several of the positions (\#3, 4, and 5). UV radiation was effective in reducing Salmonella contamination regardless of its location on tomato surfaces. Therefore, if UV treatment is applied to harvested tomatoes rolling on conveyer belts in a processing line, pathogen reduction on the tomatoes is possible. To ensure that tomatoes receive adequate UV treatments, the speed of the conveyer belt would have to be monitored. Wilson et al., (1997) reported that postharvest decay on apples was reduced when treated with UV light on a processing line. The result in this study might show lower log reduction than the previous study, but more than 3 log reduction was still observed for UV at higher dose.

\subsection{Lack of Photoreactivation of Injured Salmonella Post-UV-C Treatments}

Statistically, there was no relationshipinteraction between the UV exposure and the ability of injured Salmonella to recover after exposure to visible light. Therefore, significant differences were analyzed only among UV treatment and among visible treatment. Salmonella counts after UV radiation were significantly lower than those of controls ( $\mathrm{p}<0.05)$ (Fig. 4고). Since Salmonella population levels on tomato surfaces exposed to visible light for 3 or $5 \mathrm{~h}$ were less than those on tomatoes not exposed to visible light, no significant injury repair by photoreactivation by visible light treatments was observed. The lower Salmonella population on 
those exposed to visible light might be due to the fact that there was limited nutrient availability on the tomato surfaces or the surfaces were too dry to support recovery of Salmonella. Lang et al., (2004) found that air drying reduced recovery of E. coli O157:H7, Salmonella, and $L$. monocytogenes on the surface of tomatoes by more than 1 log, suggesting that bacterial cells may be injured or stressed during desiccationdessication.

To determine if recovery under darkness occurred, post-UV treated Salmonella contaminated tomatoes were stored in closed stainless steel containers. .(Fig. 4). Since there a lack of interaction, significant differences were analyzed only among UV treatment and among dark treatment. The results were similar to those of the visible light treatment with Salmonella levels reduced significantly $(\mathrm{p}>0.05)$ after UV treatment (Fig. 5).- The treatments of $3 \mathrm{~h}$ and $5 \mathrm{~h}$ dark exposure did not result in injury recovery.

Kuo et al., (1997) reported similar results on the lack of repair of UV damage. Their study indicated that neither $1 \mathrm{~h}$ of light exposure nor $1 \mathrm{~h}$ of dark treatment post-UV treatment significantly influenced the photoreactivation or dark repair ability of $S$. Typhimurium on shell eggs. However, photoreactivation of $S$. dysenteriae and $S$. Typhimurium was detected in a study done by Hu et al., (2012). After UV light treatment, contaminated surface water samples were exposed to a fluorescent lamp with an intensity of $0.066 \mathrm{~mW} / \mathrm{cm}^{2}$ at a wavelength of $360 \mathrm{~nm}$ for $3 \mathrm{~h}$. They noticed that photoreactivation decreased significantly with higher UV doses. Various factors may affect photoreactivation. Lindenauer \& Darby (1994) noted that the number ofinitial pyrimidine dimers formed, the amount of photolyase present, the temperature during the formation of the photoreactivation enzyme and dimer complex, and the dose of photoreactivation radiation could affect photoreactivation. Photoreactivation is dependent on the genetic and physiological state of the exposed cell (Harm, 1980).Harm (1980) also pointed out 
that "photoreactivation requires that a cell contain the photoreactivation enzyme and that the extent of repair varies with the genetic and physiological state of the cell".

\subsection{Effect of UV-C Light in Reducing Salmonella Contamination on Food Contact}

\section{Surfaces}

This study evaluated the efficacy of UV-C light in decreasing Salmonella contaminated food contact surfaces (stainless steel, HDPE, waxed cardboard, and PVC) which may be used in commercial tomato facilities. Analysis of UV light exposure for $5 \mathrm{~s}$ was done separately from the 30 s UV exposure treatment since the experiments were done on separate days. There were $\underline{\text { statistical differencesStatistically, there was interaction }(\mathrm{p} \geq \leq 0.05) \text { in Salmonella survival }}$ onbetween the UV treatment and the fourfood contact surfaces. However, the four contact surfaces were not-statistically compared to each other because one type of material is usually used for a specific application, not interchangeably. For instance, stainless steel, a widely recognized excellent material for the food industry, is usually used in the manufacture of dump tanks, processing lanes, and most equipment in tomato packinghouses (Allen et al., 2005). Meanwhile, PVC is usually used for conveyer belts or to cover roller bars which move tomatoes along the processing lines (Mukhopadhyay \& Ramaswamy, 2012). Bins made of HDPE are usually used to gather tomatoes during harvest, and waxed cardboard boxes can be used to pack tomatoes for distribution.

UV exposure for $5 \mathrm{~s}\left(3.3 \mathrm{~mJ} / \mathrm{cm}^{2}\right)$ significantly reduced Salmonella populations on all food contact surfaces (Fig. 6ㄷ). Salmonella was decreased by 2.75, 2.93, 1.39, and $1.91 \log$ CFU/coupon on SS, HDPE, WC, and PVC, respectively. Salmonella log reduction in waxed cardboard was not as great as that on other coupons. Significant differences were also observed between the $30 \mathrm{~s}$ of UV treatment and the controls (Fig. $\underline{76}$ ). Thirty seconds $\left(19.7 \mathrm{~mJ} / \mathrm{cm}^{2}\right)$ of UV 
exposure, respectively, resulted in 3.51, 4.32, 1.43, and 3.51 log reductions of Salmonella contamination on SS, HDPE, WC, and PVC. When comparing the results of coupons treated for $5 \mathrm{~s}$ and $30 \mathrm{~s}$ of UV, coupons exposed for longer time had greater Salmonella population reductions. Regardless of 5 s or 30 s of UV radiation, Salmonella populations decreased the least on waxed cardboard. Yaun et al., (2004) found that microbial populations on the surface of waxed tomatoes were greater than those on the surface of unwaxed apples after UV-C exposure. They assumed that wax applied on the surface of tomatoes might have shielded bacteria from UV radiation.

Sommers et al., (2010) obtained more than 5 log reductions of Salmonella spp., Staphylococcus aureus, and Listeria monocytogenes on electroplated and bead blasted stainless steel coupons when inoculated coupons were exposed to UV-C at a dose of $400 \mathrm{~mJ} / \mathrm{cm}^{2}$. When logs. Sommers et al. (2012) exposed stainless steel and HDPE contact surfaces contaminated with Francisella tularensis to UV-C and found that exposure to $0.5 \mathrm{~J} / \mathrm{cm}^{2}$ reduced the pathogen level by more than 4 logs. Kim et al., (2002) also reported that either intensities of 250 or 500 $\mu \mathrm{W} / \mathrm{cm}^{2}$ significantly decreased L. monocytogenes, S. Typhimurium, and E. coli O157:H7 populations on stainless steel chips. Intensity at $500 \mu \mathrm{W} / \mathrm{cm}^{2}$ for $3 \mathrm{~min}\left(90 \mathrm{~mJ} / \mathrm{cm}^{2}\right)$ reduced populations of the three pathogens by more than 4 logs. Intensity of $250 \mu \mathrm{W} / \mathrm{cm}^{2}$ for $1 \mathrm{~min}$ (15 $\mathrm{mJ} / \mathrm{cm}^{2}$ ) decreased the levels of the pathogens by 2.43-4.38 logs. This result is similar to the present findings, in which a 3.51 log reduction was achieved when stainless steel was treated for $30 \mathrm{~s}$ at $19.7 \mathrm{~mJ} / \mathrm{cm}^{2}$. Bae and Lee (2012) demonstrated that UV treatment at intensity $0.236 \pm$ $0.013 \mathrm{~mW} / \mathrm{cm}^{2}$ for $3 \mathrm{~h}$ significantly reduced S. Typhimurium, L. monocytogenes, and S. aureus on stainless steel surfaces by 3.06, 2.18, and $2.70 \log$ CFU/coupon, respectively, and S. aureus 
342 on polypropylene by 3.11 logs. They concluded that the effectiveness of the UV treatment

343 depends on the surface material. Haughton et al. (2011) achieved a 4.2 log reduction of $S$.

$344 \quad$ Enteritidis on 9 different food contact surfaces with UV levels from 0.010 to $0.192 \mathrm{~J} / \mathrm{cm}^{2}$ and

345 found significant reductions in Salmonella levels on all the surfaces.

This study indicated that UV-C light was effective in reducing Salmonella contamination

on tomatoes surfaces and various food contact surfaces. In addition, recovery of UV-C damaged

Salmonella due to possible photoreactivation or dark repair was not observed. The overall

findings of this study indicated that UV treatment is effective in reducing Salmonella contamination on food contact surfaces.

\section{Acknowledgements}

This material is based upon work supported by the National Institute of Food and

353 Agriculture, U.S. Dept. of Agriculture, under special project \#2009-51110-20161 and the

354 Georgia Agricultural Experiment Stations. Measurement of intensity of UV light was assisted by

355 Dr. Uwe Happek (Department of Physics and Astronomy, University of Georgia). Statistical

356 analysis was provided by Dr. Kim Love-Myers (Department of Statistics, University of Georgia). 


\section{References}

1. Allen, R. L., Warren, B.R., Archer, D.L., Sargent, S.A., \& Schneider, K.R. (2005). Survival of Salmonella spp. on the surfaces of fresh tomatoes and selected packing line materials. HortTechnology. 15:831-836.

2. Allende, A., \& Artes, F. (2003). UV-C radiation as a novel technique for keeping quality of fresh processed 'Lollo Rosso' lettuce. Food Res. Int. 36:739-746.

3. Allende, A., McEvoy J.L., Luo, Y., Artes, F., \& Wang, C.Y. (2006). Effectiveness of two-sided UV-C treatments in inhibiting natural microflora and extending the shelf-life of minimally processed ‘Red Oak Leaf’ lettuce. Food Microbiol. 23:241-249.

4. Bae, Y.-M., \& Lee, S.-Y. (2012). Inhibitory effects of UV treatment and a combination of UV and dry heat against pathogens on stainless steel and polypropylene surfaces. J. Food Sci. 77:M61-M64.

5. Bermydez-Aguirre, D., \& Barbosa-Canovas, G. (2013). Disinfection of selected vegetables under nonthermal treatments: Chlorine, acid citric,ultraviolet light and ozone. Food Control 29:82-90

6. Beuchat, L.R., Farber, J.M., Garrett, E.H., Harris, L.J., Parish, M.E., Suslow, T.V., \& Busta, F.F. (2001) Standardization of a method to determine the efficiacy of sanitizers in inactivating human pathogenic microorganisms on raw fruits and vegetables. J Food Prot. 64:1079-1084.

6.7. Bintsis, T., Litopoulou-Tzanetaki, E., \& Robinson, R.K. (2000). Existing and potential applications of ultraviolet light in the food industry - a critical review. J. Sci. Food Agric. 80:637-645. 
7.8. Center for Science in the Public Interest (CSPI). (2009). Analyzing foodborne outbreaks 1998 to 2007. Available at: http://cspinet.org/new/pdf/outbreakalertreport09.pdf. Accessed 10 Dec 2014.

8.9. Centers for Disease Control and Prevention (CDC). (2005). Outbreaks of Salmonella infections associated with eating Roma tomatoes -- United States and Canada, 2004. Morb. Mortal. Weekly Rep. 54:325-328.

9.10. Chun, H.H., Kim, J.Y., \& Song, K.B. (2010). Inactivation of foodborne pathogens in ready-to-eat salad using UV-C irradiation. Food Sci. Biotechnol. 19:547-551.

10.11. DeWaal, C.S., \& Bhuiya, F. (2007). Outbreaks by the numbers: fruits and vegetables 1990-2005. Available at: http://www.cspinet.org/foodsafety/IAFPPoster.pdf. Accessed 10 Dec 2014.

11.12. Doyle, M.P., \& Erickson, M.C. (2008). Summer meeting 2007 - the problems with fresh produce: an overview. J. Appl. Microbiol. 105:317-330.

12.13. Escalona, V.H., Aguayo, E., Martinez-Hernandez, G.B., \& Artes, F. (2010). UV-C doses to reduce pathogen and spoilage bacterial growth in vitro and in baby spinach. Postharvest Biol. Technol. 56:223-231.

14. Hadjok, C., Mittal, G.S., and Warriner, K. (2008) Inactivation of human pathogens and spoilage bacteria on the surface and internalized within fresh produce by using a combination of ultraviolet light and hydrogen peroxide. J Appl Microbiol. 104:10141024.

13.15. Hallmich, C., \& Gehr, R. (2010). Effect of pre- and post-UV disinfection conditions on photoreactivation of fecal coliforms in wastewater effluents. Water Res. 44:2885-2893. 
14.16. Harm, W. (1980). Biological effects of ultraviolet radiation. Cambridge University Press, New York, NY.

17. Haughton, P.N., Lyng, J.G., Cronin, D.A., Morgan, D.J., Fanning, S., \& Whyte, P. (2011) Efficacy of UV light treatment for the microbiological decontamination of chicken, associated packaging, and contact surfaces. J. Food Prot. 74:565-572.

15.18. Hedberg, C.W., Angulo, F.J., White, K.E., Langkop, C.W., Schell, W.L., Stobierski, M.G., Schuchat, A., Besser, J.M., Dietrich, S., Helsel, L., Griffin, P.M., McFarland, J.W., Osterholm, M.T., \& Invest., T. (1999). Outbreaks of salmonellosis associated with eating uncooked tomatoes: implications for public health. Epidemiol. Infect. 122:385-393.

16.19. Hu, X.X., Geng, S.J., Wang, X.J., \& C. Hu., C. (2012). Inactivation and photorepair of enteric pathogenic microorganisms with ultraviolet irradiation. Environ. Eng. Sci. 29:549-553.

17.20. Kim, T., Silva, J.L., \& Chen, T.C. (2002). Effects of UV irradiation on selected pathogens in peptone water and on stainless steel and chicken meat. J. Food Prot. 65:1142-1145.

18.21. Kuo, F.L., Rucke, S.C., \& Carey, J.B. (1997). UV irradiation of shell eggs: effect on populations of aerobes, molds, and inoculated Salmonella typhimurium. J. Food Prot. 60:639-643.

19.22. Lang, M.M., Beuchat, L.R., \& Harris, L.J. (2004). Evaluation of inoculation method and inoculum drying time for their effects on survival and efficiency of recovery of Escherichia coli O157:H7, Salmonella, and Listeria monocytogenes inoculated on the surface of tomatoes. J. Food Prot. 67:732-741. 
20.23. Lindenauer, K.G., \& Darby, J.L. (1994). Ultraviolet disinfection of wastewater: effect of dose on subsequent photoreactivation. Water Res. 28:805-817.

21.24. Liu, J., Stevens, C., Khan, V.A., Lu, J.Y., Wilson, C.L., Adeyeye, O., Kabwe, M.K., Pusey, P.L., E. Chalutz, E., Sultana, T., \& Droby, S. (1993). Application of ultraviolet-C light on storage rots and ripening of tomatoes. J. Food Prot. 56:868-872.

22.25. Manzocco, L., Da Pieve, S., Bertolini, A., Bartolomeoli, I., Maifreni, M., Vianello, A., \& Nicoli, M.C. (2011a). Surface decontamination of fresh-cut apple by UV-C light exposure: Effects on structure, colour and sensory properties. Postharvest Biol. Technol. 61:165-171.

23.26. Manzocco, L., Da Pieve, S., \& Maifreni, M. (2011b). Impact of UV-C light on safety and quality of fresh-cut melon. Innovat. Food Sci. Emerg. Technol. 12:13-17.

24.27. Mukhopadhyay, S. \& Ramaswamy, R. (2012). Application of emerging technologies to control Salmonella in foods: A review. Food Res. Int. 45:666-677.

25.28. Mukhopadhyay, S., Ukuku, V., Fan, X., \& Juneja,V. (2013). Efficacy of integrated treatment of UV light and low-dose gamma irradiation on inactivation of Escherichia coli 0157:H7 and Salmonella enterica on grape tomatoes. J. Food Sci. 78:M1049-M1055

26.29. Olaimat, A.N., \& Holley, R.A. (2012). Factors influencing the microbial safety of fresh produce: A review. Food Microbiol. 32:1-19.

27.30. Shechmeister, I.L. (1976). Sterilization by ultraviolet radiations, p. 522-561. In S.S. Block (ed.), Disinfection, sterilization, and preservation. Lea \& Febiger, Philadelphia, PA.

28.31. Sizer, C.E., \& Balasubramaniam, V.M. (1999). New intervention processes for minimally processed juices. Food Technol. 53:64-67. 
29.32. Sommer, R., Lhotsky, M., Haider, T., \& Cabaj, A. (2000). UV inactivation, liquidholding recovery, and photoreactivation of Escherichia coli $\mathrm{O} 157$ and other pathogenic Escherichia coli strains in water. J. Food Prot. 63:1015-1020.

33. Sommers, C.H., Scullen, O.J., Paoli, G.C., and Bhaduri, S. (2012) Inactivation of Francisella tularensis Utah-112 on food and food contact surfaces by ultraviolet light. $J$ Food Process Technol. S11-002.

30.34. Sommers, C. H., Sites, J.E. \& Musgrove, M. (2010). Ultraviolet light (254 nm) inactivation of pathogens on foods and stainless steel surfaces. J. Food Saf. 30:470-479.

31.35. Song, H.J., Choi, D.W., \& Song, K.B. (2011). Effect of aqueous chlorine dioxide and UV-C treatment on the microbial reduction and color of cherry tomatoes. Hort. Environ. Biotechnol. 52:488-493.

32.36. Tosa, K., \& Hirata, T. (1999). Photoreactivation of enterohemorrhagic Escherichia coli following UV disinfection. Water Res. 33:361-366.

33.37. Williams, L.L, Yang, W.W, English, T., English, N., Johnson, J.U., Rababah, T. Khatiwada, J. (2012). Disinfection of Salmonella spp. on tomato surface by pulsed ultraviolet light and selected sanitizers. Internat. J. Food Engin. doi:10.1515/15563758.2063

34.38. Wilson, C.L., El-Ghaouth, A., Upchurch, B., Stevens, C., Khan, V., Droby, S., \& Chalutz, E. (1997). Using an on-line UV-C apparatus to treat harvested fruit for controlling postharvest decay. HortTechnology. 7:278-282.

35.39. Wong, E., Linton, R.H., \& D E. Gerrard, D.E. (1998). Reduction of Escherichia coli and Salmonella senftenberg on pork skin and pork muscle using ultraviolet light. Food Microbiol. 15:415-423.37. 
36.40. Yaun, B.R., Sumner, S.S., Eifert, J.D., \& Marcy, J.E. (2004). Inhibition of pathogens on 470 fresh produce by ultraviolet energy. Int. J. Food Microbiol. 90:1-8.

38. Yousef, A.E., \& Marth, E.H. (1988). Inactivation of Listeria monocytogenes by ultraviolet energy. J. Food Sci. 53:571-573.

37.41. Yun, J., Yan, R., Fan, X., Gurtler, J. \& Phillips, J. (2013). Fate of E. coli O155:H7, Salmonella spp. and potential surrogate bacteria on apricot fruit, following exposure to UV-C light. Internat. J. Food Microbiol. 166:356-363.

38.42. Zimmer, J.L., \& Slawson, R. M. (2002). Potential repair of Escherichia coli DNA following exposure to UV radiation from both medium- and low-pressure UV sources used in drinking water treatment. Appl. Environ. Microbiol. 68:3293-3299. 


\section{List of Figures}

Figure 1. Placement of inoculated tomatoes in the UV chamber. Marked areas indicate the positioning of the inoculated surface in relation to the UV-C light (as viewed from top).

Figure 2. Survival of Salmonella on tomato surfaces after UV light treatment at an intensity of $743.6 \mu \mathrm{W} / \mathrm{cm}^{2}$ for 0 to $300 \mathrm{~s}$. Values with different letters were significantly different $(\mathrm{p}<0.05)$

Figure $\underline{3} z$. Survival of Salmonella on 6 different locations on the tomato surfaces after treatment of UV light at intensity of $651 \mu \mathrm{W} / \mathrm{cm}^{2}$ from 0 to $180 \mathrm{~s}$. Salmonella contamination positions on tomato surfaces: 1 - directly exposed to UV source; 2 and 5 - on equatorial plane facing reflective side wall of UV protective shield; 3 - on equatorial plane facing tomatoes; 4 and 6 - on equatorial plane facing reflective end walls of UV protective shield. Figure 43 . Survival of Salmonella on tomato surfaces after exposure to visible light for 0 , 3, and 5 h at each UV light treatment of $0,30,60$, and 120 s.

Figure 54 . Survival of Salmonella on tomato surfaces after storage in the dark for 0 , 3, and $5 \mathrm{~h}$ at each UV light treatment of $0,30,60$, and $120 \mathrm{~s}$.

Figure 6 5. Survival of Salmonella on food contact surfaces after UV light treatment at an intensity of $656 \mu \mathrm{W} / \mathrm{cm}^{2}$ for 0 and 5 s. SS: stainless steel; HDPE: high density polyethylene; WC: waxed cardboard; PVC: polyvinyl chloride. Values within each contact surface type with different letters were significantly different $(\mathrm{p}<0.05)$.

Figure 76 . Survival of Salmonella on food contact surfaces after UV light treatment at an intensity of $656 \mu \mathrm{W} / \mathrm{cm}^{2}$ for 0 and 30 s. SS: stainless steel; HDPE: high density polyethylene; WC: waxed cardboard; PVC: polyvinyl chloride. Values within each contact surface type with different letters were significantly different $(\mathrm{p}<0.05)$. 
Fig. 1

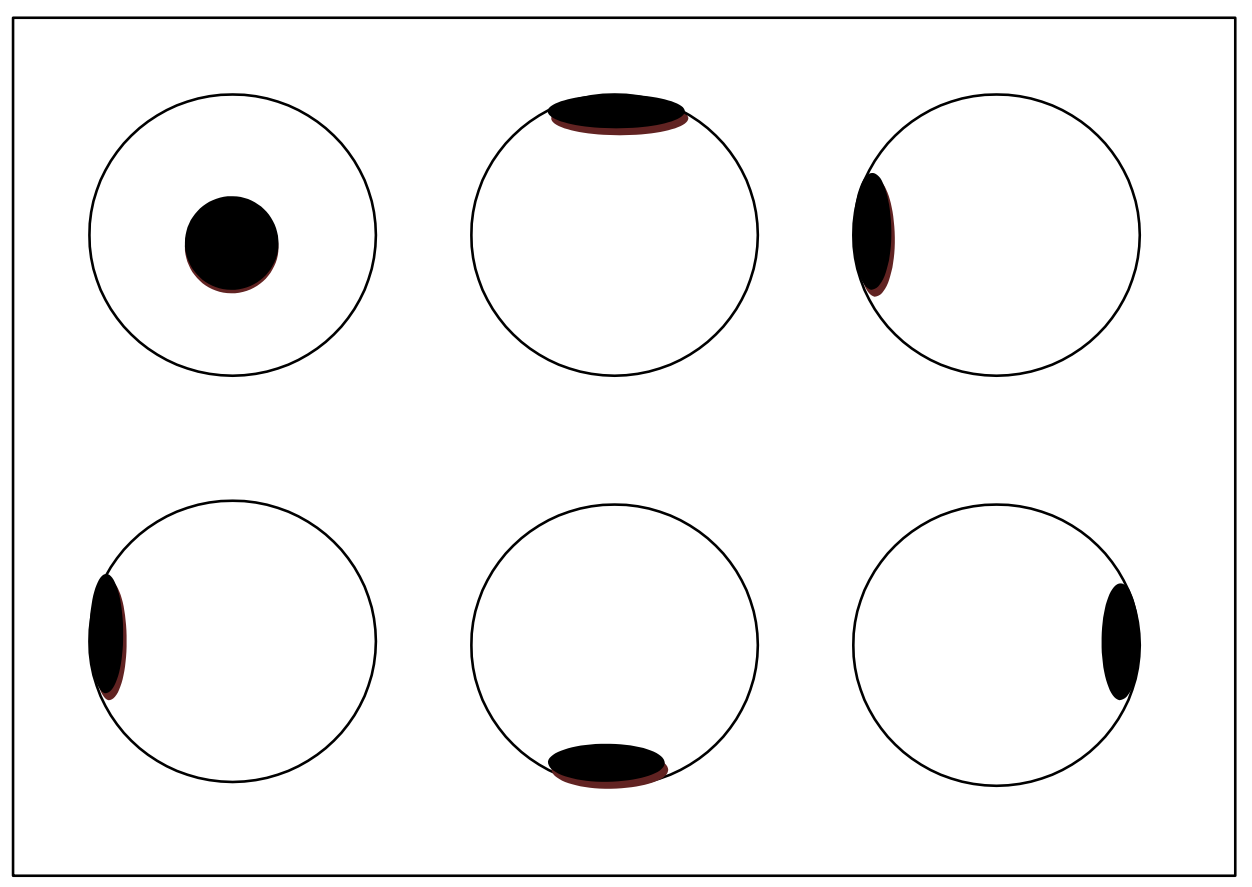


Fig. 2

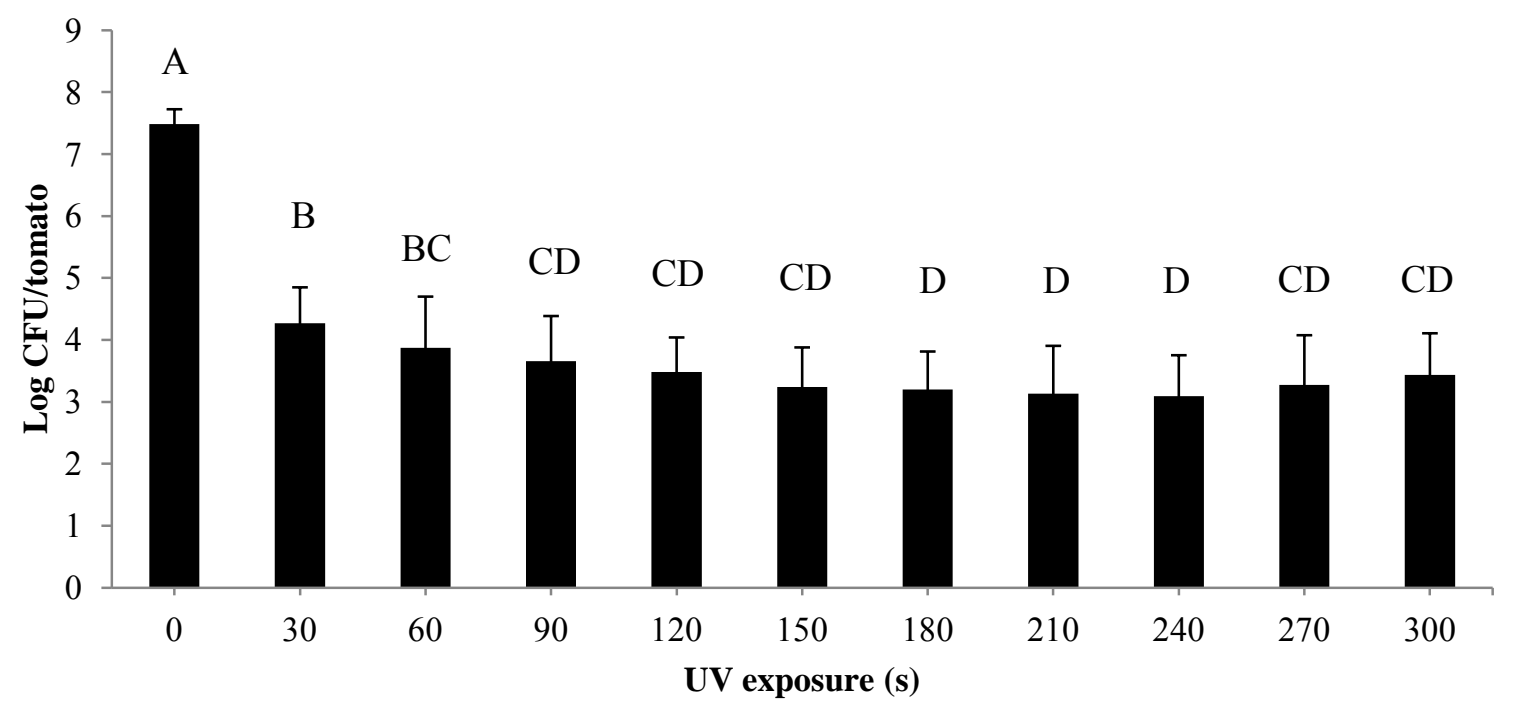


Fig. 3

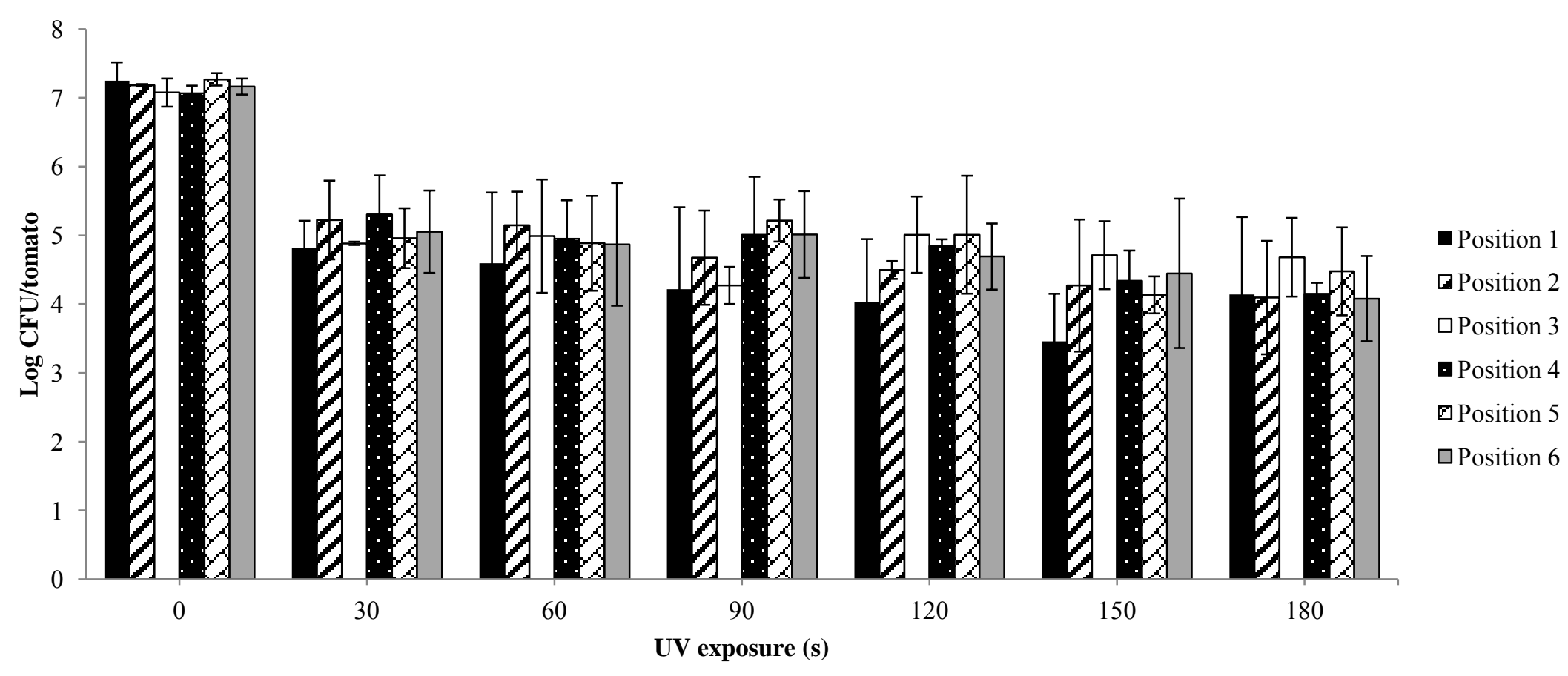


Fig. 4

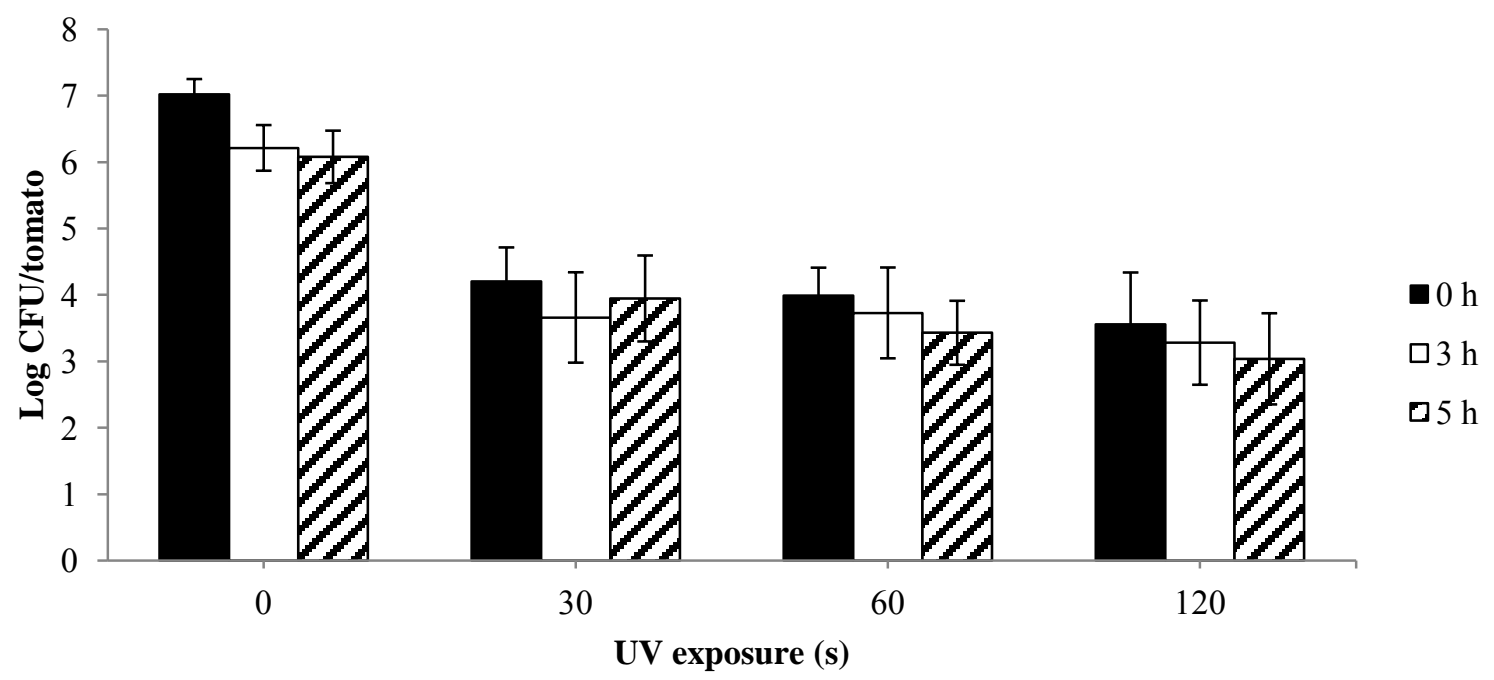


Fig. 5

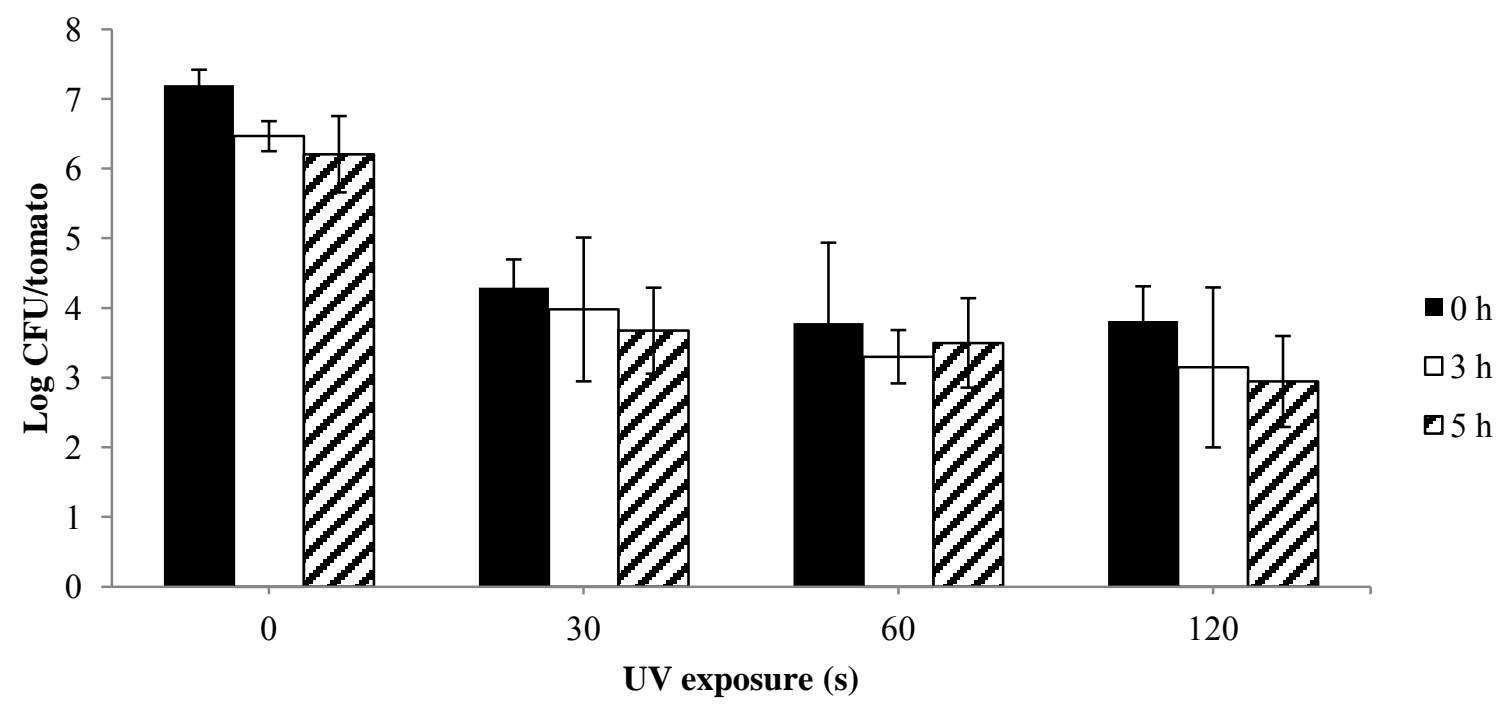


Fig. 6

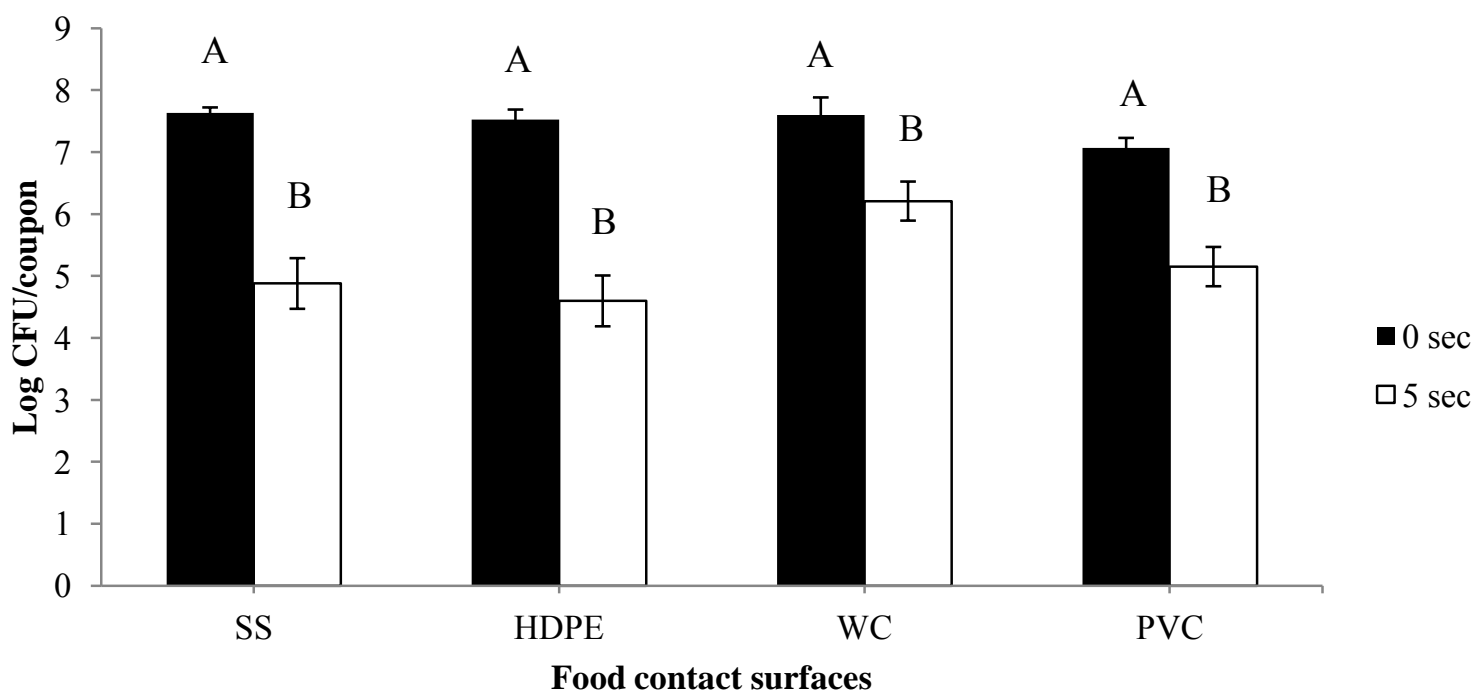


Fig. 7

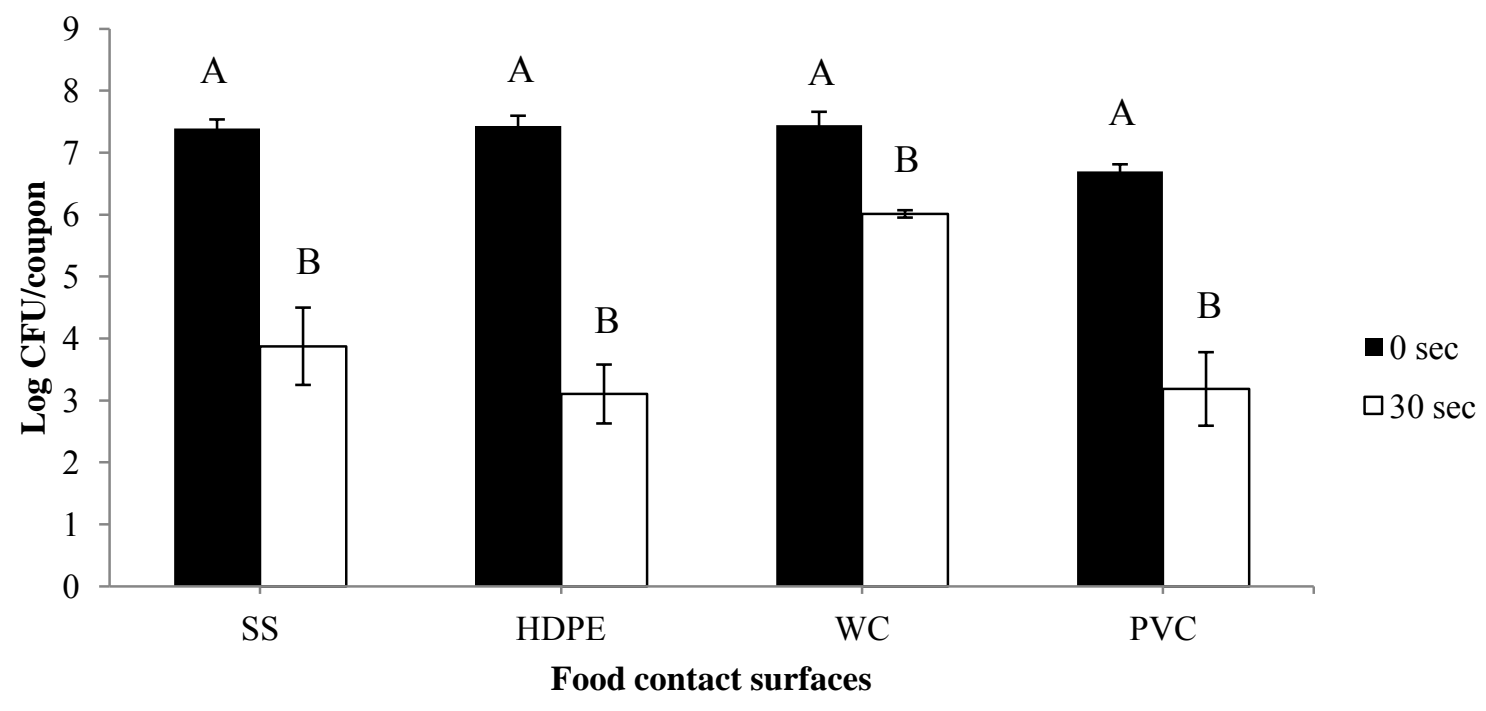

